\title{
Evidence for oxygen vacancies movement during wake-up in ferroelectric hafnium oxide
}

S. Starschich, S. Menzel, and U. Böttger

Citation: Appl. Phys. Lett. 108, 032903 (2016); doi: 10.1063/1.4940370

View online: http://dx.doi.org/10.1063/1.4940370

View Table of Contents: http://aip.scitation.org/toc/apl/108/3

Published by the American Institute of Physics

\section{Articles you may be interested in}

Ferroelectricity and ferroelectric resistive switching in sputtered $\mathrm{Hf0} .5 \mathrm{Zr0.5O2}$ thin films Appl. Phys. Lett. 108, 232905232905 (2016); 10.1063/1.4953461

A thermally robust and thickness independent ferroelectric phase in laminated hafnium zirconium oxide Appl. Phys. Lett. 6, 095123095123 (2016); 10.1063/1.4964300

Fully ALD-grown TiN/Hf0.5Zr0.5O2/TiN stacks: Ferroelectric and structural properties Appl. Phys. Lett. 109, 192903192903 (2016); 10.1063/1.4966219

Ferroelectric properties of full plasma-enhanced ALD TiN/La:HfO2/TiN stacks Appl. Phys. Lett. 108, 242905242905 (2016); 10.1063/1.4953787 


\title{
Evidence for oxygen vacancies movement during wake-up in ferroelectric hafnium oxide
}

\author{
S. Starschich, ${ }^{1}$ S. Menzel, ${ }^{2}$ and U. Böttger ${ }^{1}$ \\ ${ }^{1}$ Institut für Werkstoffe der Elektrotechnik 2, RWTH Aachen University, Sommerfeldstraße 24, D-52074 \\ Aachen, Germany \\ ${ }^{2}$ Peter Grünberg Institut 7, Forschungszentrum Jülich GmbH, D-52425 Jülich, Germany
}

(Received 3 December 2015; accepted 9 January 2016; published online 21 January 2016)

\begin{abstract}
The wake-up effect which is observed in ferroelectric hafnium oxide is investigated in yttrium doped hafnium oxide prepared by chemical solution deposition. It can be shown that not the amount of cycles but the duration of the applied electrical field is essential for the wake-up. Temperature dependent wake-up cycling in a range of $-160^{\circ} \mathrm{C}$ to $100^{\circ} \mathrm{C}$ reveals a strong temperature activation of the wake-up, which can be attributed to ion rearrangement during cycling. By using asymmetrical electrodes, resistive valence change mechanism switching can be observed coincident with ferroelectric switching. From the given results, it can be concluded that redistribution of oxygen vacancies is the origin of the wake-up effect. (C) 2016 AIP Publishing LLC.
\end{abstract}

[http://dx.doi.org/10.1063/1.4940370]

The recently discovered unconventional lead free ferroelectric hafnium oxide is a promising candidate for future high density, low power, and nonvolatile memory applications. ${ }^{1,2}$ Further fields of application are piezoelectric and pyroelectric sensors; pyroelectric energy harvesting, in particular, is of great interest. ${ }^{3-5}$ Although up to now there has been great progress in understanding the origin of the ferroelectricity, the wake-up effect is not fully understood yet. Wake-up means the initial increase of the remanent polarization during cycling, where mostly a constricted hysteresis is observed in the pristine state, which opens up after cycling. The effect was observed for several ALD (atomic layer deposition) prepared samples doped with $\mathrm{Si}^{6}{ }^{6} \mathrm{Gd},{ }^{7}$ and $\mathrm{Sr}^{8}$ and for yttrium doped samples prepared by sputtering ${ }^{9}$ and by chemical solution deposition (CSD). ${ }^{10}$ Therefore, it seems to be a material property rather than originating from the deposition technique. Understanding the underlying effect of the wake-up is essential for the application of hafnium oxide in electronic devices. As a possible explanation, Zhou and Lomenzo proposed that a depletion layer is formed between the bottom electrode and the hafnium oxide due to oxidation of the TiN during the deposition of the hafnium oxide. ${ }^{6,11}$ This effect can be excluded for our samples due to the use of platinum electrodes. Park et al. assumed a structural phase transition from the tetragonal to the orthorhombic phase during wake-up for films thinner than $8 \mathrm{~nm} .{ }^{12}$ Schenk et al. showed that in a pristine sample two distinct maxima in the $I(V)$ curve are biased against each other and merge together during cycling. ${ }^{13}$ Furthermore, they assumed that this internal bias could be attributed to mobile ions, which are rearranged after wake-up cycling.

Besides ferroelectric switching, bipolar resistive switching in $\mathrm{HfO}_{\mathrm{x}}$ thin films sandwiched between two metal (M) electrodes has been frequently reported. ${ }^{14-20}$ Thereby, the resistance of the $\mathrm{M} / \mathrm{HfO}_{\mathrm{x}} / \mathrm{M}$ devices is changed between a high resistive and a low resistive state by application of positive and negative voltage signals. The resistive switching effect is based on the movement of oxygen vacancies within the thin film, which leads to a valence change in the Hf cations and a change in the local conductivity. ${ }^{21-23}$ Thus, this switching mechanism is also called valence change mechanism (VCM) ${ }^{24}$ In general, an electroforming step is required before stable resistive switching is achieved. During this electroforming step, the oxide thin film is reduced by extraction of oxygen via one of the electrodes, and an oxygen-deficient filament is formed. ${ }^{24-26}$ Alternatively, a sub-stoichiometric oxide layer can be deposited in order to achieve forming-free switching behavior. ${ }^{27}$

In this letter, we report our results on wake-up investigations of CSD prepared yttrium doped hafnium oxide. The frequency and temperature dependency of the wake-up is investigated. By using asymmetric electrodes, resistive VCM switching can be shown in quasi static $I(V)$ curves. From the given results, the wake-up can be clearly related to oxygen vacancy movement during the applied electrical fields.

The 5.2\% yttrium doped hafnium oxide prepared by CSD and the platinum electrodes used in this work were fabricated as described in Ref. 28. The titanium nitride electrode for the asymmetric stack was reactively sputtered from a 6 in. titanium target at room temperature using $10 \%$ nitrogen in an argon atmosphere. The $P(V)$ and wake-up measurements were performed with a Keithley 4200 , whereby the $P(V)$ curves were calculated from the measured $I(V)$ curves. The quasi static $I(V)$ measurements for investigation of the resistive switching were performed with a Keithley 6430 at a fixed current range. If not mentioned otherwise, the $P(V)$ curves were measured at a frequency of $1 \mathrm{kHz}$ on device areas of $2.5 \times 10^{-4} \mathrm{~cm}^{2}$.

At first, the frequency dependence of the wake-up is investigated using a $40 \mathrm{~nm}$ thick hafnium oxide layer with platinum top and bottom electrodes. Therefore, the wake-up is performed at different frequencies as shown in Figure 1. The cycling and measurement sequence is depicted in the inset of Figure 1(b). A strong frequency dependence of the remanent polarization can be observed (Figure 1(a)). At higher wake-up frequencies, a higher amount of cycles is 

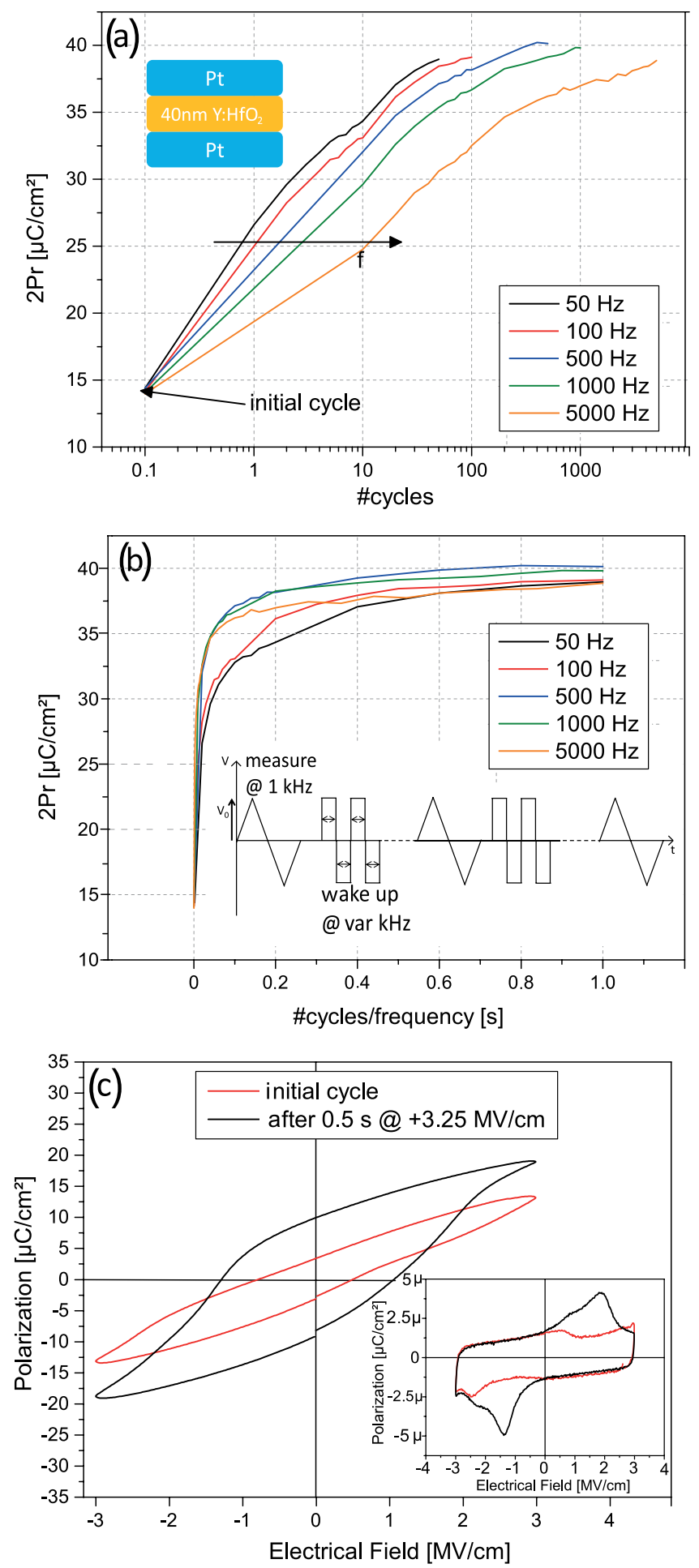

FIG. 1. (a) The double remanent polarization $2 \mathrm{P}_{\mathrm{r}}$ depending on the number of performed cycles at varying frequencies is shown. For higher wake up frequencies, a higher amount of cycles is necessary to reach the same polarization. (b) $2 \mathrm{P}_{\mathrm{r}}$ is shown over the duration of the applied electrical field (\#cycles/frequency). The inset shows the cycling and measurement sequence performed on the samples. The hysteresis is measured at $1 \mathrm{kHz}$, and the wake up cycle frequency is varied between $50 \mathrm{~Hz}$ and $5 \mathrm{kHz}$ at $3.25 \mathrm{MV} / \mathrm{cm}$. (c) Wake-up with a positive dc voltage for $0.5 \mathrm{~s}$. A split up of the hysteresis is visible, although the maximum polarization as observed for cycling wakeup is not reached.

necessary to reach the same remanent polarization. By plotting the remanent polarization over the duration of the applied field (\#cycles/frequency), the curves at different frequencies show the same dependence. Thus, not the amount of cycles but the duration of the applied electrical field is essential for the wake-up. That means that 50 cycles at $50 \mathrm{~Hz}$ show the same results as $5 \mathrm{k}$ cycles at $5 \mathrm{kHz}$. Further cycling leads to an ongoing degradation of the device. Therefore, a maximum time of $1 \mathrm{~s}$ was applied during wake-up for these results. Figure 1(c) shows the wake-up with a dc voltage applied for $0.5 \mathrm{~s}$. It can be seen that also with a dc voltage a wake-up can be realized, although the remanent polarization is lower compared to the cycling wake-up. The results of the temperature dependent wake-up are depicted in Figure 2. A strong temperature dependence can be observed with a much faster wake-up at higher temperatures. Furthermore, the maximum remanent polarization after cycling is higher at elevated temperatures. In the initial cycle, the hysteresis is pinched as observed by Schenk et al. for strontium doped hafnium oxide, ${ }^{29}$ which is indicated by two distinct current maxima at each polarity. During cycling, the two peaks are merging together to one single current peak as shown in Figure 2. Further cycling at lower temperatures does not lead to a significant increase of remanent polarization, whereas by increasing the electrical field also higher polarizations can be achieved at lower temperatures (data not shown here). The most noticeable difference for the different temperatures is the remanent polarization during the first cycle. For elevated temperatures, the prepolarization cycle is sufficient to achieve a higher remanent polarization compared to the lowest temperatures after the full wake-up. This strong temperature dependence indicates the presence of ions that are mobile at the applied electrical field. At elevated temperatures, a higher amount of trapped ions is mobile resulting in a higher remanent polarization. Due to the trivalent yttrium ion replacing the tetravalent hafnium in the crystal, a high amount of oxygen vacancies is present in the oxide layer. ${ }^{30}$ Therefore, most probably, mobile oxygen vacancies in hafnium oxide are causing the observed wake-up effect. It should be mentioned that as described in the introduction also isovalent dopants ( $\mathrm{Si}, \mathrm{Zr}$ ) show wake-up behavior where no oxygen vacancies are created due to the dopant. But there are also different possibilities to create oxygen vacancies in the oxide like, for example, extraction of oxygen via one of the electrodes. ${ }^{24-26}$ To prove our assumption, the layer stack is modified by a $10 \mathrm{~nm}$ thick TiN layer between the hafnium oxide and the top platinum electrode. Furthermore, the thickness of the hafnium oxide is reduced to $15 \mathrm{~nm}$. An asymmetrical stack is used because it is known to be preferable for VCM resistive switching. ${ }^{14,16,18,31}$ Figure 3 shows on the left side the initial $P(V)$ cycle and the layer stack in the inset. For the wake-up, $100 \mathrm{k}$ cycles are applied at a frequency of 10 $\mathrm{kHz}$ at 3.6 MV/cm (Figure 3(a)). In contrast to the thicker films without titanium nitride shown before, more cycles are necessary to achieve the maximum polarization, which was also observed by Hoffmann et al. ${ }^{32}$ By applying a negative quasi-static triangle signal with the same maximum electrical field of $3.6 \mathrm{MV} / \mathrm{cm}$ as for the wake-up, an electroforming sets in as known from resistive VCM switching of hafnium oxide $^{14-20}$ (Figure 3(b)). During forming into the high resistive state, a current compliance of $10 \mu \mathrm{A}$ is applied to protect the device from permanent breakdown. After forming, the characteristic $I(V)$ curves with stable resistive switching are observed. Again, a current compliance is required to protect 


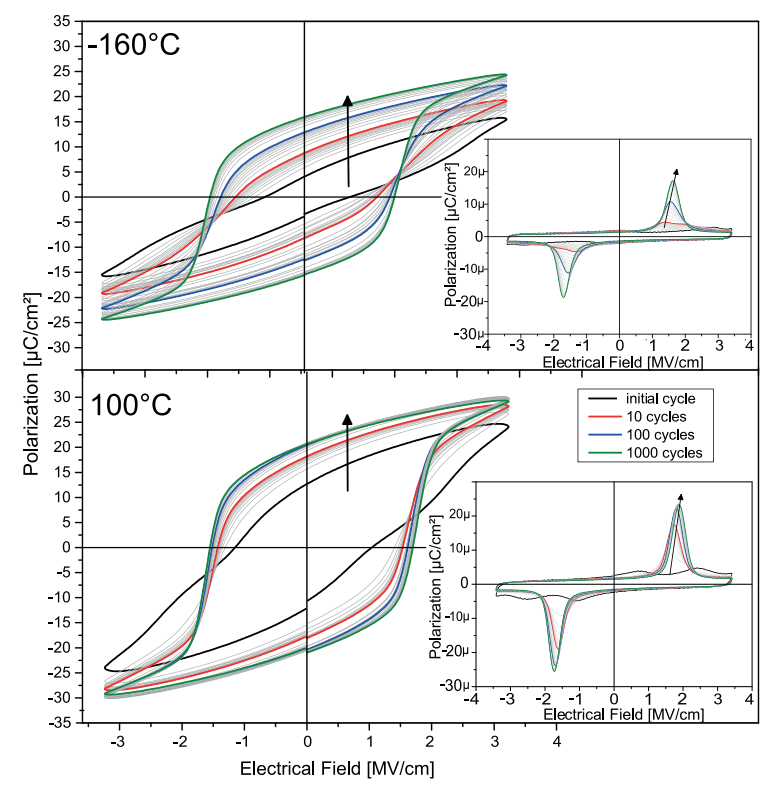

the device from breakdown during switching. VCM resistive switching is attributed to the movement of oxygen vacancies during the forming step as well as during the resistive switching after a stable plug has been formed..$^{21-23}$ As depicted in Figure 3, the electrical field during wake-up is as high as the forming field. The difference is that during the forming step the voltage is applied for a longer time. This could lead to an incorporation of additional vacancies via the electrodes facilitating the filament formation. Alternatively, this long time is required to agglomerate the existing oxygen vacancies in a filamentary region. After the filament has formed, the resistive switching effect will dominate the device behavior. Due to the low resistance of the filament, any ferroelectric response would be masked. A further important observation is that the forming conditions do not change after wake-up. Therefore, after the wake-up the oxygen vacancies are still sufficiently mobile and are redistributed during each performed cycle. This can lead to a device breakdown during cycling as observed by several groups. ${ }^{11,32,33}$ Mobile vacancies are crucial for the investigation of the endurance behavior of the device.

To prove the hypothesis that mobile oxygen vacancies explain the wake-up effect, the ion velocity under the experimental conditions is calculated. As a first approximation, the velocity of the oxygen vacancies can be calculated using the Mott-Gurney equation

$$
v=a f \exp \left(\frac{W_{0}}{K_{B} T}\right) \sinh \left(\frac{z a}{2 K_{B} T} E\right),
$$

where $a$ is the hopping distance, $f$ is the attempt frequency, $W_{0}$ is the activation energy, $z$ is the charge of the ion, and $E$ is the electric field. For a first approximation, the values measured by Zafar et al. are used $(a=0.25 \mathrm{~nm} ; f=1$ $\times 10^{14} \mathrm{~s}^{-1}$; and $\left.W_{0}=0.6 \mathrm{eV}\right){ }^{34}$ Furthermore, the following values are used: $z=2$ (double positively charged oxygen

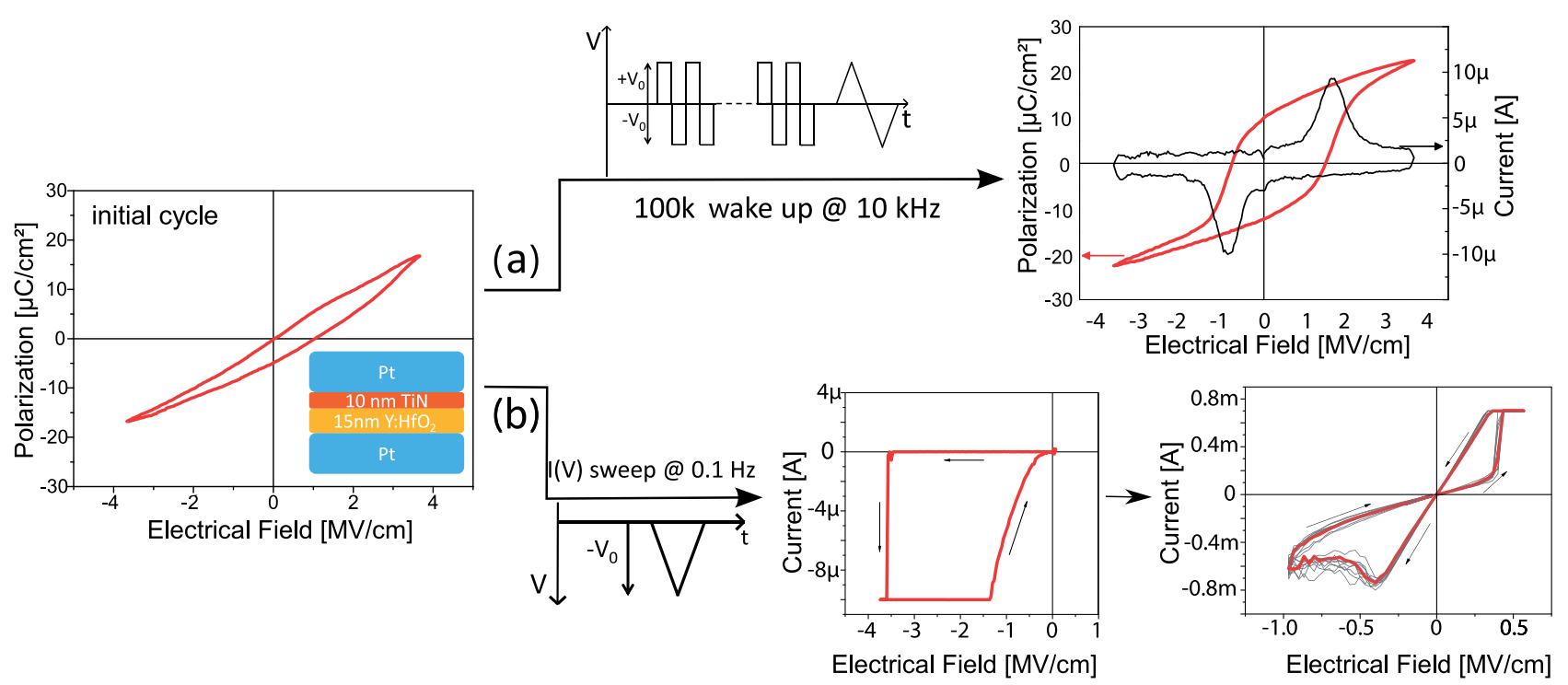

FIG. 3. The left side shows the initial P(V) cycle and the modified layer stack. The wake up as described before is depicted in the upper path (a). By applying a triangle voltage to an uncycled device at a much lower frequency of $0.1 \mathrm{~Hz}$, an electroforming step takes place with stable resistive VCM switching after the forming (lower path (b)). 
vacancies); $T=295 \mathrm{~K}$; and $E=3.25 \mathrm{MV} / \mathrm{cm}$ (the applied electrical field). Calculating with the given parameters, a velocity of $1.3 \times 10^{-5} \mathrm{~m} / \mathrm{s}$ results. By using a wake-up frequency of $1 \mathrm{kHz}$, the voltage is applied for $0.5 \mathrm{~ms}$ for each polarity during each cycle. This results in a migration distance of $6.5 \mathrm{~nm}$ during each cycle. Therefore, it seems plausible that oxygen vacancies are sufficiently mobile during the duration of the applied electrical field.

From this observation, it can be concluded that during the wake-up the oxygen vacancies are redistributed in the hafnium oxide layer, leading to a stabilization of the ferroelectric phase. This approves the assumptions of Zhou and Schenk mentioned in the introduction. Also for further investigations of the fatigue and ferroelectric switching behavior, the mobility of the oxygen vacancies should be kept in mind.

In summary, the wake-up effect was investigated in detail for CSD prepared ferroelectric hafnium oxide. It was shown that not the amount of cycles but the duration of the applied electrical field is essential for the formation of the ferroelectric hysteresis. A strong temperature dependence for the wake-up was found, indicating ion movement during an applied electrical field. The shown resistive VCM switching clearly indicates that during the wake-up a redistribution of oxygen vacancies takes place leading to the stabilization of the ferroelectric phase.

This work was funded by German Research Foundation (Deutsche Forschungsgemeinschaft) within the scope of the project "Inferox" (Project No. BO 1629/10-1).

${ }^{1}$ T. S. Boescke, J. Mueller, D. Braeuhaus, U. Schroeder, and U. Boettger, "Ferroelectricity in hafnium oxide thin films," Appl. Phys. Lett. 99, 102903 (2011).

${ }^{2}$ J. Mueller, P. Polakowski, S. Mueller, and T. Mikolajick, "Ferroelectric hafnium oxide based materials and devices: Assessment of current status and future prospects," ECS J. Solid State Sci. Technol. 4, N30-N35 (2015).

${ }^{3}$ M. H. Park, H. J. Kim, Y. J. Kim, T. Moon, K. D. Kim, and C. S. Hwang, "Toward a multifunctional monolithic device based on pyroelectricity and the electrocaloric effect of thin antiferroelectric $\mathrm{Hf}_{\mathrm{x}} \mathrm{Zr}_{1-\mathrm{x}} \mathrm{O}_{2}$ films," Nano Energy 12, 131-140 (2015).

${ }^{4}$ M. H. Park, Y. H. Lee, H. J. Kim, Y. J. Kim, T. Moon, K. Do Kim, J. Mueller, A. Kersch, U. Schroeder, T. Mikolajick, and C. S. Hwang, "Ferroelectricity and antiferroelectricity of doped thin $\mathrm{HfO}_{2}$-based films," Adv. Mater. 27, 1811-1831 (2015).

${ }^{5}$ M. H. Park, H. J. Kim, Y. J. Kim, T. Moon, K. D. Kim, and C. S. Hwang, "Thin $\mathrm{Hf}_{\mathrm{x}} \mathrm{Zr}_{1-\mathrm{x}} \mathrm{O}_{2}$ films: A new lead-free system for electrostatic supercapacitors with large energy storage density and robust thermal stability," Adv. Energy Mater. 4, 1400610 (2014).

${ }^{6}$ D. Zhou, J. Xu, Q. Li, Y. Guan, F. Cao, X. Dong, J. Mueller, T. Schenk, and U. Schroeder, "Wake-up effects in Si-doped hafnium oxide ferroelectric thin films," Appl. Phys. Lett. 103, 192904 (2013).

${ }^{7}$ S. Mueller, C. Adelmann, A. Singh, S. Van Elshocht, U. Schroeder, and T. Mikolajick, "Ferroelectricity in Gd-doped $\mathrm{HfO}_{2}$ thin films," ECS J. Solid State Sci. Technol. 1, N123-N126 (2012).

${ }^{8}$ T. Schenk, S. Mueller, U. Schroeder, R. Materlik, A. Kersch, M. Popovici, C. Adelmann, S. Van Elshocht, and T. Mikolajick, "Strontium doped hafnium oxide thin films: Wide process window for ferroelectric memories," in ESSDERC (2013).

${ }^{9}$ T. Olsen, U. Schroeder, S. Mueller, A. Krause, D. Martin, A. Singh, J. Mueller, M. Geidel, and T. Mikolajick, "Co-sputtering yttrium into hafnium oxide thin films to produce ferroelectric properties," Appl. Phys. Lett. 101, 082905-1-082905-4 (2012).

${ }^{10} \mathrm{~S}$. Starschich, D. Griesche, T. Schneller, R. Waser, and U. Boettger, "Chemical solution deposition of ferroelectric yttrium-doped hafnium oxide films on platinum electrodes," Appl. Phys. Lett. 104, 202903 (2014).
${ }^{11}$ P. D. Lomenzo, Q. Takmeel, C. Zhou, C. M. Fancher, E. Lambers, N. G. Rudawski, J. L. Jones, S. Moghaddam, and T. Nishida, "TaN interface properties and electric field cycling effects on ferroelectric Si-doped $\mathrm{HfO}_{2}$ thin films," J. Appl. Phys. 117, 134105 (2015).

${ }^{12}$ M. H. Park, H. J. Kim, Y. J. Kim, Y. H. Lee, T. Moon, K. D. Kim, S. D. Hyun, and C. S. Hwang, "Study on the size effect in $\mathrm{Hf}_{0.5} \mathrm{Zr}_{0.5} \mathrm{O}_{2}$ films thinner than $8 \mathrm{~nm}$ before and after wake-up field cycling," Appl. Phys. Lett. 107, 192907 (2015).

${ }^{13}$ T. Schenk, M. Hoffmann, J. Ocker, M. Pesic, T. Mikolajick, and U. Schroeder, "Complex internal bias fields in ferroelectric hafnium oxide," ACS Appl. Mater. Interfaces 7, 20224-20233 (2015).

${ }^{14}$ S. Brivio, J. Frascaroli, and S. Spiga, "Role of metal-oxide interfaces in the multiple resistance switching regimes of Pt/HfO2/TiN devices," Appl. Phys. Lett. 107, 023504-1-023504-5 (2015).

${ }^{15}$ H. Y. Lee, P. S. Chen, T. Y. Wu, Y. S. Chen, C. C. Wang, P. J. Tzeng, C. H. Lin, F. Chen, C. H. Lien, and M.-J. Tsai, "Low power and high speed bipolar switching with a thin reactive Ti buffer layer in robust $\mathrm{HfO}_{2}$ based RRAM," IEEE Int. Electron Dev. Meet. 2008, $1-4$.

${ }^{16}$ M. Sowinska, T. Bertaud, D. Walczyk, S. Thiess, P. Calka, L. Alff, C. Walczyk, and T. Schroeder, "In-operando hard X-ray photoelectron spectroscopy study on the impact of current compliance and switching cycles on oxygen and carbon defects in resistive switching $\mathrm{Ti} / \mathrm{HfO}_{2} / \mathrm{TiN}$ cells," J. Appl. Phys. 115, 204509 (2014).

${ }^{17}$ L. Zhao, H. Chen, S. Wu, Z. Jiang, S. Yu, T. Hou, H. P. Wong, and Y. Nishi, "Multi-level control of conductive nano-filament evolution in $\mathrm{HfO}_{2}$ ReRAM by pulse-train operations," Nanoscale 6, 5698-5702 (2014).

${ }^{18}$ L. Goux, X. P. Wang, Y. Y. Chen, L. Pantisano, N. Jossart, B. Govoreanu, J. A. Kittl, M. Jurczak, L. Altimime, and D. J. Wouters, "Roles and effects of TiN and $\mathrm{Pt}$ electrodes in resistive-switching $\mathrm{HfO}_{2}$ systems," Electrochem. Solid-State Lett. 14, H244 (2011).

${ }^{19}$ P. Gonon, M. Mougenot, C. Vallee, C. Jorel, V. Jousseaume, H. Grampeix, and F. El Kamel, "Resistance switching in $\mathrm{HfO}_{2}$ metal-insulator-metal devices,” J. Appl. Phys. 107, 074507 (2010).

${ }^{20}$ M. Lanza, G. Bersuker, M. Porti, E. Miranda, M. Nafria, and X. Aymerich, "Resistive switching in hafnium dioxide layers: Local phenomenon at grain boundaries," Appl. Phys. Lett. 101, 193502 (2012).

${ }^{21}$ J. J. Yang, M. D. Pickett, X. Li, D. A. A. Ohlberg, D. R. Stewart, and R. S. Williams, "Memristive switching mechanism for metal/oxide/metal nanodevices," Nat. Nanotechnol. 3, 429-433 (2008).

${ }^{22}$ S. Larentis, F. Nardi, S. Balatti, D. C. Gilmer, and D. Ielmini, "Resistive switching by voltage-driven ion migration in bipolar RRAM-Part II: Modeling," IEEE Trans. Electron Devices 59, 2468-2475 (2012).

${ }^{23}$ A. Marchewka, B. Roesgen, K. Skaja, H. Du, C.-L. Jia, J. Mayer, V. Rana, R. Waser, and S. Menzel, "Nanoionic resistive switching memories: On the physical nature of the dynamic reset process," Adv. Electron. Mater. (published online 2015).

${ }^{24}$ R. Waser, R. Dittmann, G. Staikov, and K. Szot, "Redox-based resistive switching memories-Nanoionic mechanisms, prospects, and challenges," Adv. Mater. 21, 2632-2663 (2009).

${ }^{25}$ J. J. Yang, F. Miao, M. D. Pickett, D. A. A. Ohlberg, D. R. Stewart, C. N. Lau, and R. S. Williams, "The mechanism of electroforming of metal oxide memristive switches," Nanotechnology 20, 215201 (2009).

${ }^{26} \mathrm{E}$. Abbaspour, S. Menzel, and C. Jungemann, "The role of the interface reactions in the electroforming of redox-based resistive switching devices using KMC simulations," in 2015 International Conference on Simulation of Semiconductor Processes and Devices (SISPAD), Washington D.C., USA (2015), pp. 293-296.

${ }^{27}$ S. U. Sharath, T. Bertaud, J. Kurian, E. Hildebrandt, C. Walczyk, P. Calka, P. Zaumseil, M. Sowinska, D. Walczyk, A. Gloskovskii, T. Schroeder, and L. Alff, "Towards forming-free resistive switching in oxygen engineered $\mathrm{HfO}_{2-\mathrm{x}}$," Appl. Phys. Lett. 104, 063502 (2014).

${ }^{28}$ S. Starschich, D. Griesche, T. Schneller, and U. Böttger, "Chemical solution deposition of ferroelectric hafnium oxide for future lead free ferroelectric devices," ECS J. Solid State Sci. Technol. 4, P419-P423 (2015).

${ }^{29}$ T. Schenk, U. Schroeder, M. Pesic, M. Popovici, Y. V. Pershin, and T. Mikolajick, "Electric field cycling behavior of ferroelectric hafnium oxide," ACS Appl. Mater. Interfaces 6, 19744-19751 (2014).

${ }^{30}$ L. Zhao, S.-W. Ryu, A. Hazeghi, D. Duncan, B. Magyari-Köpe, and Y. Nishi, "Dopant selection rules for extrinsic tunability of $\mathrm{HfO}_{\mathrm{x}}$ RRAM characteristics: A systematic study," in Proceedings of the 2013 Symposium on VLSI Technology (2013).

${ }^{31}$ A. Marchewka, R. Waser, and S. Menzel, "Physical simulation of dynamic resistive switching in metal oxides using a Schottky contact barrier 
model," in 2015 International Conference on Simulation of Semiconductor Processes and Devices (SISPAD), Washington D.C., USA (2015), pp. 297-300.

${ }^{32}$ M. Hoffmann, U. Schroeder, T. Schenk, T. Shimizu, H. Funakubo, O. Sakata, D. Pohl, M. Drescher, C. Adelmann, R. Materlik, A. Kersch, and T. Mikolajick, "Stabilizing the ferroelectric phase in doped hafnium oxide," J. Appl. Phys. 118, 072006 (2015).
${ }^{33}$ M. H. Park, H. J. Kim, Y. J. Kim, T. Moon, K. D. Kim, Y. H. Lee, S. D. Hyun, and C. S. Hwang, "Study on the internal field and conduction mechanism of atomic layer deposited ferroelectric $\mathrm{Hf}_{0.5} \mathrm{Zr}_{0.5} \mathrm{O}_{2}$ thin films," J. Mater. Chem. C 3, 6291-6300 (2015).

${ }^{34}$ S. Zafar, H. Jagannathan, L. F. Edge, and D. Gupta, "Measurement of oxygen diffusion in nanometer scale $\mathrm{HfO}_{2}$ gate dielectric films," Appl. Phys. Lett. 98, 152903 (2011). 